\title{
A versatile route to benzodiazocine and spiropyran derivatives through chalcones
}

\author{
JOHNSON GEORGE ${ }^{\mathrm{a}}$, SAURABH SINGH ${ }^{\mathrm{a}}$, \\ RAHUL JOSHI $^{\mathrm{a}}$ and RAMCHAND T PARDASANI ${ }^{\mathrm{b}, *}$ \\ ${ }^{a}$ Department of Chemistry, University of Rajasthan, Jaipur 302 055, India \\ ${ }^{b}$ Department of Chemistry, Central University of Rajasthan, Bandarsindri, Ajmer 305 802, India \\ e-mail: rtpardasani@curaj.ac.in
}

MS received 2 November 2012; revised 26 March 2013; accepted 25 April 2013

\begin{abstract}
Syntheses of 3-(phenyl)-benzo[b]thiophene [2, 3-d][1,2] benzodiazocine derivatives have been accomplished by the reaction of 3-phenacylidine-2-benzo[b]thiophene-2-ones with $o$-phenylene diamine. The photolytic reaction with trans-stilbene resulted in the exclusive formation of spiro $\left\{2^{\prime}, 5^{\prime}, 6^{\prime}\right.$-triphenyl-2H-pyran$\left.4^{\prime}, 3\right\}$-benzo[b]thiophene-2-one derivatives. Theoretical calculations have been performed to study the mechanism and stereoselectivity of products. Good yield and broad scope of usable substrates of industrial relevance are other prominent features of the present methodologies.
\end{abstract}

Keywords. Thioisatin; chalcones; benzodiazocines; spiropyrans; DFT calculations; transition state.

\section{Introduction}

The thiophene ring system is probably the most wellknown heterocycle, a common and important feature of various natural products and medicinal agents. ${ }^{1}$ Its diketo derivative, benzo[b]thiophene-2,3-dione has an important applications in synthetic organic chemistry. ${ }^{2}$ Incorporation of seven or eight-membered rings into the basic structural moiety may lead to pharmacologically active molecules. ${ }^{3}$ A perusal of literature revealed that no chemistry of thioisatin-based benzodiazocine derivatives has been explored. For this purpose, the chalcones have been proved as potential building blocks for the synthesis of various heterocyclic systems and have shown a wide variety of biological activities. ${ }^{4}$

During the last three decades, the photochemistry of organic molecules has grown into an important and pervasive branch of organic chemistry. Photochemical cycloaddition provides one of the most efficient and versatile methods for the construction of carbo- and heterocycles of different sizes with high atom economy. Chow and co-workers have described the photocycloadditions of $\alpha$-diketones, ${ }^{5}$ yet the fascinating photochemistries of derivatives of benzo[b]thiophenes have remained largely unexplored. To make a contribution to these ongoing research fields connected with our interest in the synthesis of spiropyan compounds we here report a $[4+2]$ photocycloaddition pathway

*For correspondence for spiropyran heterocycles. Motivated by our recent works on 'stereoselectivity in $[3+2]$ cycloaddition of azomethine ylides ${ }^{6}$ and photochemistry of quinones ${ }^{7}$ and their $[2+2 / 3+2]$ photocycloadditions', ${ }^{8}$ we have examined the feasibility of phenacylidines (chalcones) derived from thioisatin and a variety of ketones as building blocks for the construction of novel heterocyclic frameworks of possible industrial relevance and the results are presented here.

\section{Experimental}

\subsection{General procedures}

Melting points were determined in an open glass capillary and are uncorrected. The solvents were purified by standard procedures. ${ }^{9}$ The IR spectra were recorded on Nicolet Magna IR TM model 550 in $\mathrm{KBr}$ pellets. The ${ }^{1} \mathrm{H}$ and ${ }^{13} \mathrm{C}$ NMR spectra were obtained on a JEOL AL-300 instrument at 300 and $75 \mathrm{MHz}$ using $\mathrm{CDCl}_{3}$ or DMSO- $\mathrm{d}_{6}$ as solvent and tetramethylsilane (TMS) as an internal standard. Chemical shifts are given in $\delta \mathrm{ppm}$. Elemental analyses were performed by Perkin Elmer series C, H, N and S analyser-2400. Photochemical irradiation was conducted in a Heber multilamp photoreactor (Model: HML-COMPACT-SW-MW-LW888) consisting of a quartz/borosilicate reactor surrounded by $8+8+8$ numbers of UV lamps ( 8 lamps can be operated at a time: either at $254 \mathrm{~nm} / 312 \mathrm{~nm} / 365 \mathrm{~nm}$ 
with $8+8+8$ separate controls) permanently fixed inside the reaction chamber with built-in highly polished anodized aluminium reflector ( $85 \%$ reflection) so that UV rays are focused at the centre where the sample being kept. The reaction chamber is covered by reactor house which does not allow leakage of any UV irradiation. Thin-layer chromatography (TLC) was performed on alumina foil on Merck's Kiesel gel $60 \mathrm{~F}_{254}$ sheets, visualization was achieved at ultra fluorescence on an Indian Equipment Corporation equipment, IEC-312 at $354 \mathrm{~nm}$. Column chromatography was carried over silica gel 60-120 mesh as adsorbent, using solvents of rising polarity.

\subsection{Computational details}

All computations were performed via different theoretical methods by using the Gaussian 03 suite of programs. ${ }^{10}$ The optimization of the geometries and population analysis were carried out in detail by DFTB3LYP/6-31G* molecular orbital calculations. Harmonic vibration frequencies of all stationary points have been computed to characterize them as energy minima (all frequencies are real) or transition states (one and only one imaginary frequency). An imaginary frequency has been obtained for each transition state which substantiates the actual formation of the transition state. Intrinsic reaction coordinates (IRC) calculations have been carried out to confirm the reaction pathway and the transition states.

2.2a Synthesis of chalcones: Acetophenone or its derivatives $2(0.04 \mathrm{~mol})$ were added consecutively with stirring to thioisatin $1(0.04 \mathrm{~mol})$ in the presence of catalytic amount of diethylamine. The mixture was refluxed for $8-10 \mathrm{~h}$ and the hydroxyl derivative obtained as precipitate was filtered off and recrystallized from ethanol. Then these derivatives undergo dehydration in the presence of $\mathrm{AcOH}-\mathrm{HCl}$ mixture to give chalcones (3a-h).

2.2b Physical and analytical data chalcone derivative (3a): Yellow crystalline solid. Yield: $65 \%$. m.p: 173-175 ${ }^{\circ}$. IR (KBr), 3040-3000 (Ar-H), 1720, 1645 $(\mathrm{C}=\mathrm{O}), 630(-\mathrm{C}-\mathrm{S}) \mathrm{cm}^{-1} \cdot{ }^{1} \mathrm{H} \mathrm{NMR} 300 \mathrm{MHz} ; \mathrm{CDCl}_{3}$; $\left.\mathrm{Me}_{4} \mathrm{Si}\right) ; \delta$ 7.71-7.26 (m, 8H, $\left.\mathrm{Ar}-\mathrm{H}\right), 4.52(\mathrm{~s}, 1 \mathrm{H}$, $\mathrm{C}=\mathrm{CH}), 2.42\left(\mathrm{~s}, 3 \mathrm{H}, \mathrm{CH}_{3}\right) ;{ }^{13} \mathrm{C} \mathrm{NMR}\left(75 \mathrm{MHz} ; \mathrm{CDCl}_{3}\right.$; $\left.\mathrm{Me}_{4} \mathrm{Si}\right): \delta 24.25\left(\mathrm{CH}_{3}\right), 114.08(\mathrm{C}=\mathrm{CH}), 140.00$ 125.50 (Ar-C), $163.66(\mathrm{C}=\mathrm{O})$. Found: $\mathrm{C}, 72.16 ; \mathrm{H}$, 4.65; S, 11.18\%. Calcd for $\mathrm{C}_{17} \mathrm{H}_{12} \mathrm{O}_{2} \mathrm{~S}: \mathrm{C}, 72.83 ; \mathrm{H}$, $4.31 ; \mathrm{S}, 11.44 \%$.

\subsection{Synthesis of benzodiazocine derivatives}

The $o$-phenylene diamine $(0.5 \mathrm{mmol})$ was added to the solution of chalcone $3(0.5 \mathrm{mmol})$ in absolute ethanol $(25 \mathrm{ml})$ at room temperature. Progress of the reaction was monitored on TLC plate. After $21 \mathrm{~h}$ colour of the reaction mixture changed from dark red to light which indicated completion of the reaction. The compound separated out was filtered, washed with cold petroleum ether and recrystallized from petroleum ether-chloroform mixture (3:1).

\subsection{Physical and analytical data of benzodiazocine derivatives}

2.4a 3-(Phenyl)-6-methyl benzo[b]thiophene[2,3-d][1,2] benzodiazocine (4a): White powder. Yield: $56 \%$. m.p: $227^{\circ} \mathrm{C}$. IR (KBr), 3050-3000 (Ar-H), 1610, 1575 $(\mathrm{C}=\mathrm{N}), 1550-1430(\mathrm{C}=\mathrm{C}) \mathrm{cm}^{-1} .{ }^{1} \mathrm{H} \mathrm{NMR} 300 \mathrm{MHz}$; $\left.\mathrm{CDCl}_{3} ; \mathrm{Me}_{4} \mathrm{Si}\right) ; \delta$ 7.72-6.82 (m, 12H, Ar-H), $5.6(\mathrm{~s}$, $1 \mathrm{H}, \mathrm{C}=\mathrm{CH}), 2.4\left(\mathrm{~s}, 3 \mathrm{H}, \mathrm{CH}_{3}\right) ;{ }^{13} \mathrm{C} \mathrm{NMR}(75 \mathrm{MHz}$; $\left.\mathrm{CDCl}_{3} ; \mathrm{Me}_{4} \mathrm{Si}\right): \delta 21.70\left(\mathrm{CH}_{3}\right), 124.20-145.90(\mathrm{Ar}-$ C), $155.50(\mathrm{C}=\mathrm{N}), 159.20(\mathrm{C}=\mathrm{N})$. Found: C, 78.52; H, 4.67; N, 8.10; S, 8.93\%. Calcd for $\mathrm{C}_{23} \mathrm{H}_{16} \mathrm{~N}_{2} \mathrm{~S}: \mathrm{C}, 78.38$; H, 4.58; N, 7.95; S, 9.10\%.

$2.4 \mathrm{~b}$ 3-(2-Methyl phenyl)-6-methyl benzo[b]thiophene [2,3-d][1,2]benzodiazocine (4b): Fade brown solid. Yield: $62 \%$. m.p: $198-200^{\circ} \mathrm{C}$. IR (KBr), 3070-3010 $(\mathrm{Ar}-\mathrm{H}), 1510,1565(\mathrm{C}=\mathrm{N}), 1530-1490(\mathrm{C}=\mathrm{C}) \mathrm{cm}^{-1}$. ${ }^{1} \mathrm{H}$ NMR $\left(300 \mathrm{MHz} ; \mathrm{CDCl}_{3} ; \mathrm{Me}_{4} \mathrm{Si}\right) ; \delta$ 7.60-6.80 (m, $11 \mathrm{H}, \mathrm{Ar}-\mathrm{H}), 5.7(\mathrm{~s}, 1 \mathrm{H}, \mathrm{C}=\mathrm{CH}), 2.4\left(\mathrm{~s}, 3 \mathrm{H}, \mathrm{CH}_{3}\right)$, $2.5\left(\mathrm{~s}, 3 \mathrm{H}, \mathrm{CH}_{3}\right) ;{ }^{13} \mathrm{C}$ NMR $\left(75 \mathrm{MHz} ; \mathrm{CDCl}_{3} ; \mathrm{Me}_{4} \mathrm{Si}\right)$ : $\delta 20.30\left(\mathrm{CH}_{3}\right), 22.70\left(\mathrm{CH}_{3}\right), 123.60-146.20(\mathrm{Ar}-\mathrm{C})$, $154.60(\mathrm{C}=\mathrm{N}), 158.70(\mathrm{C}=\mathrm{N})$. Found: $\mathrm{C}, 78.20 ; \mathrm{H}$, 4.88; N, 7.81; S, 8.69\% Calcd for $\mathrm{C}_{24} \mathrm{H}_{18} \mathrm{~N}_{2} \mathrm{~S}$ : C, 78.66; H, 4.95; N, 7.64; S, 8.75\%.

\section{4c 3-(2-Pyridine)-6-methylbenzo[b]thiophene [2,3-d]}

[1,2]benzodiazocine $(\mathbf{4 c )}$ : Fade yellow powder. Yield: 63\%. m.p: $204-207^{\circ} \mathrm{C}$. IR (KBr), 3050-3000 $(\mathrm{Ar}-\mathrm{H}), 1620,1580(\mathrm{C}=\mathrm{N}), 1530-1450(\mathrm{C}=\mathrm{C}) \mathrm{cm}^{-1}$. ${ }^{1} \mathrm{H}$ NMR $\left(300 \mathrm{MHz} ; \mathrm{CDCl}_{3} ; \mathrm{Me}_{4} \mathrm{Si}\right) ; \delta 7.72-6.85(\mathrm{~m}$, $11 \mathrm{H}, \mathrm{Ar}-\mathrm{H}), 5.5(\mathrm{~s}, 1 \mathrm{H}, \mathrm{C}=\mathrm{CH}), 2.4\left(\mathrm{~s}, 3 \mathrm{H}, \mathrm{CH}_{3}\right)$; ${ }^{13} \mathrm{CNMR}\left(75 \mathrm{MHz} ; \mathrm{CDCl}_{3} ; \mathrm{Me}_{4} \mathrm{Si}\right): \delta 20.90\left(\mathrm{CH}_{3}\right)$, 126.20-148.50 (Ar-C), $155.50(\mathrm{C}=\mathrm{N}), 158.60(\mathrm{C}=\mathrm{N})$. Found: C, 74.35; H, 4.20; N, 11.75; S, 9.20\%. Calcd for $\mathrm{C}_{22} \mathrm{H}_{15} \mathrm{~N}_{3} \mathrm{~S}$ : C, 74.76; H, 4.28; N, 11.89; S, 9.07\%.

2.4d 3-(2-Thiophene)-6-methylbenzo[b]thiophene[2,3-d] [1,2]benzodiazocine (4d): Brown solid. Yield: 59\%. m.p: $196^{\circ} \mathrm{C}$ (decompose). IR (KBr), 3050-2990 (Ar$\mathrm{H}), 1580,1540(\mathrm{C}=\mathrm{N}), 1510-1475(\mathrm{C}=\mathrm{C}) \mathrm{cm}^{-1} \cdot{ }^{1} \mathrm{H}$ 
NMR $\left(300 \mathrm{MHz} ; \mathrm{CDCl}_{3} ; \mathrm{Me}_{4} \mathrm{Si}\right) ; \delta$ 8.25-6.86 (m, $10 \mathrm{H}, \mathrm{Ar}-\mathrm{H}), 5.50(\mathrm{~s}, 1 \mathrm{H}, \mathrm{C}=\mathrm{CH}), 2.30\left(\mathrm{~s}, 3 \mathrm{H}, \mathrm{CH}_{3}\right)$, ${ }^{13} \mathrm{CNMR}\left(75 \mathrm{MHz} ; \mathrm{CDCl}_{3} ; \mathrm{Me}_{4} \mathrm{Si}\right): \delta 21.30\left(\mathrm{CH}_{3}\right)$, 123.50-147.80 ( $\mathrm{Ar}-\mathrm{C}), 156.60(\mathrm{C}=\mathrm{N}), 160.20(\mathrm{C}=\mathrm{N})$. Found: C, 69.95; H, 3.86; N, 7.92; S, 17.96\%. Calcd for $\mathrm{C}_{21} \mathrm{H}_{14} \mathrm{~N}_{2} \mathrm{~S}_{2}: \mathrm{C}, 70.36 ; \mathrm{H}, 3.94 ; \mathrm{N}, 7.81 ; \mathrm{S}, 17.89 \%$.

2.4e 3-(2-Phenyl)-benzo[b]thiophene [2,3-d][1,2] benzodiazocine (4e): Grey solid. Yield: 57\%. m.p: $241^{\circ} \mathrm{C}$ (decompose). IR (KBr), 3050-3000 (Ar-H), 1570, $1550(\mathrm{C}=\mathrm{N}), 1535-1515(\mathrm{C}=\mathrm{C}) \mathrm{cm}^{-1} .{ }^{1} \mathrm{H}$ NMR $\left(300 \mathrm{MHz} ; \mathrm{CDCl}_{3} ; \mathrm{Me}_{4} \mathrm{Si}\right) ; \delta$ 7.90-6.85 (m, 13H, Ar$\mathrm{H}), 5.6(\mathrm{~s}, 1 \mathrm{H}, \mathrm{C}=\mathrm{CH}) ;{ }^{13} \mathrm{C}$ NMR $\left(75 \mathrm{MHz}, \mathrm{CDCl}_{3}\right.$, $\left.\mathrm{Me}_{4} \mathrm{Si}\right): \delta$ 126.00-144.00 (Ar-C), $155.90 \quad(\mathrm{C}=\mathrm{N})$, $160.50(\mathrm{C}=\mathrm{N})$. Found: $\mathrm{C}$, 77.86; H, 4.26; N, 8.13; S, 9.39\%. Calcd for $\mathrm{C}_{22} \mathrm{H}_{14} \mathrm{~N}_{2} \mathrm{~S}: \mathrm{C}, 78.08 ; \mathrm{H}, 4.17 ; \mathrm{N}$, $8.28 ; \mathrm{S}, 9.48 \%$.

2.4f 3-(2-Methyl phenyl)-benzo[b]thiophene[2,3-d][1,2] benzodiazocine $(\mathbf{4 f})$ : Purple coloured powder. Yield: 52\%. m.p: $203-205^{\circ} \mathrm{C}$. IR (KBr), 3040-3010 ( $\left.\mathrm{Ar}-\mathrm{H}\right)$, 1580, $1555(\mathrm{C}=\mathrm{N}), 1530-1520(\mathrm{C}=\mathrm{C}) \mathrm{cm}^{-1} .{ }^{1} \mathrm{H} \mathrm{NMR}$ $\left(300 \mathrm{MHz} ; \mathrm{CDCl}_{3} ; \mathrm{Me}_{4} \mathrm{Si}\right) ; \delta$ 7.84-6.96 (m, 12H, Ar$\mathrm{H}), 5.5(\mathrm{~s}, 1 \mathrm{H}, \mathrm{C}=\mathrm{CH}), 2.3\left(\mathrm{~s}, 3 \mathrm{H}, \mathrm{CH}_{3}\right) ;{ }^{13} \mathrm{C} \mathrm{NMR}$ $\left(75 \mathrm{MHz} ; \mathrm{CDCl}_{3} ; \mathrm{Me}_{4} \mathrm{Si}\right.$ ): $\delta$ 128.00-145.00 (Ar-C), $156.75(\mathrm{C}=\mathrm{N}), 159.20(\mathrm{C}=\mathrm{N})$. Found: $\mathrm{C}, 77.98 ; \mathrm{H}$, 4.32; N, 7.75; S, 9.03\%. Calcd for $\mathrm{C}_{23} \mathrm{H}_{16} \mathrm{~N}_{2} \mathrm{~S}: \mathrm{C}, 78.38$; $\mathrm{H}, 4.58 ; \mathrm{N}, 7.95 ; \mathrm{S}, 9.10 \%$.

2.4g 3-(2-Pyridine)benzo[b]thiophene [2,3-d][1,2] benzodiazocine $(\mathbf{4 g})$ : Grey powder. Yield: $65 \%$. m.p: $175-178^{\circ} \mathrm{C}$. IR (KBr) 3050-3000 (Ar-H), 1600, 1540 $(\mathrm{C}=\mathrm{N}), 1520-1490(\mathrm{C}=\mathrm{C}) \mathrm{cm}^{-1} .{ }^{1} \mathrm{H}$ NMR $(300 \mathrm{MHz}$; $\left.\mathrm{CDCl}_{3} ; \mathrm{Me}_{4} \mathrm{Si}\right) ; \delta$ 7.90-6.60 (m, 12H, Ar-H), $5.5(\mathrm{~s}$, $1 \mathrm{H}, \mathrm{C}=\mathrm{CH}) ;{ }^{13} \mathrm{C}$ NMR $\left(75 \mathrm{MHz} ; \mathrm{CDCl}_{3} ; \mathrm{Me}_{4} \mathrm{Si}\right)$ :

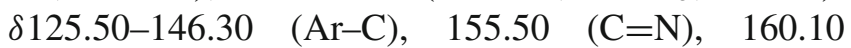
$(\mathrm{C}=\mathrm{N})$. Found: C, 74.23; H, 3.94; N, 12.30; S, 9.33\%. Calcd for $\mathrm{C}_{21} \mathrm{H}_{13} \mathrm{~N}_{3} \mathrm{~S}$ : C, 74.31; H, 3.86; N, 12.38; S, $9.41 \%$.

2.4h 3-(2-Thiophene)benzo[b]thiophene [2,3-d][1,2] benzodiazocine (4h): White powder. Yield: $63 \%$. m.p: $171-174^{\circ} \mathrm{C}$ (decompose). IR (KBr), 3040-2980 $(\mathrm{Ar}-\mathrm{H}), 1570,1540(\mathrm{C}=\mathrm{N}), 1520-1485(\mathrm{C}=\mathrm{C}) \mathrm{cm}^{-1}$. ${ }^{1} \mathrm{H}$ NMR $\left(300 \mathrm{MHz} ; \mathrm{CDCl}_{3} ; \mathrm{Me}_{4} \mathrm{Si}\right) ; \delta 8.20-6.90(\mathrm{~m}$, $11 \mathrm{H}, \mathrm{Ar}-\mathrm{H}), 5.50(\mathrm{~s}, 1 \mathrm{H}, \mathrm{C}=\mathrm{CH}) ;{ }^{13} \mathrm{C}$ NMR $(75 \mathrm{MHz}$; $\mathrm{CDCl}_{3} ; \mathrm{Me}_{4} \mathrm{Si}$ ): $\delta$ 125.60-148.90 (Ar-C), 156.90 $(\mathrm{C}=\mathrm{N}), 160.30(\mathrm{C}=\mathrm{N})$. Found: $\mathrm{C}, 69.66 ; \mathrm{H}, 3.43 ; \mathrm{N}$, 8.20; S, 18.53\%. Calcd for $\mathrm{C}_{20} \mathrm{H}_{12} \mathrm{~N}_{2} \mathrm{~S}_{2}$ : C, 69.74; H, $3.51 ; \mathrm{N}, 8.13 ; \mathrm{S}, 18.62 \%$.

\subsection{Synthesis of spiropyran derivatives}

A solution of chalcone $3(5 \mathrm{mmol})$ and trans-stilbene $(5 \mathrm{mmol})$ in dry benzene $(25 \mathrm{ml})$ was placed in quartz tube and irradiated at $312 \mathrm{~nm}$ under nitrogen atmosphere using Heber multilamp photoreactor for $16 \mathrm{~h}$ with magnetic stirring. After completion of the reaction which was monitored by thin-layer chromatography, the reaction mixture was concentrated under reduced pressure, furnished the product which was purified by column chromatography over silica gel.

2.5a Spiro $\left\{2^{\prime}, 5^{\prime}, 6^{\prime}\right.$-triphenyl-2H-pyran-4',3\}-5-methylbenzo [b] thiophene-2-one (5a): Brown solid. Yield: 56\%. m.p: $221-224^{\circ} \mathrm{C}$. IR (KBr), 3010-3043 (Ar-H), 1720 $(\mathrm{C}=\mathrm{O}), 1610 \quad(\mathrm{C}=\mathrm{C}), 608 \quad(\mathrm{C}-\mathrm{S}) \quad \mathrm{cm}^{-1} \cdot{ }^{1} \mathrm{H}$ NMR (300 MHz; $\left.\mathrm{CDCl}_{3} ; \mathrm{Me}_{4} \mathrm{Si}\right) ; \delta 6.72-7.35$ (m, 8H, Ar-H), $5.29(\mathrm{~d}, 1 \mathrm{H}, \mathrm{CH}), 4.98(\mathrm{~s}, 1 \mathrm{H}, \mathrm{CH}), 4.01(\mathrm{~d}, 1 \mathrm{H}, \mathrm{CH})$, $2.21\left(\mathrm{~s}, 3 \mathrm{H}, \mathrm{CH}_{3}\right) ;{ }^{13} \mathrm{C} \mathrm{NMR}\left(75 \mathrm{MHz} ; \mathrm{CDCl}_{3} ; \mathrm{Me}_{4} \mathrm{Si}\right)$ : $\delta 20.9\left(\mathrm{CH}_{3}\right), 58.90(\mathrm{CH}), 72.25(\mathrm{CH}), 81.60$ (spiro C), 126.50-149.10 (Ar-C), $192.90(\mathrm{C}=\mathrm{O})$. Found: C, 81.15; H, 5.56; S, 6.78\%. Calcd for $\mathrm{C}_{31} \mathrm{H}_{24} \mathrm{O}_{2} \mathrm{~S}$ : C, $80.84 ; \mathrm{H}, 5.25 ; \mathrm{S}, 6.96 \%$.

2.5b Spiro $\left\{2^{\prime}\right.$ (o-toluene) $5^{\prime}, 6^{\prime}$-diphenyl-2H-pyran-4',3\}-5methylbenzo[b] thiophene-2-one (5b): Greenish yellow solid. Yield: $51 \%$. m.p: $169^{\circ} \mathrm{C}$. IR (KBr), 30003010 (Ar-H), $1740(\mathrm{C}=\mathrm{O}), 1605(\mathrm{C}=\mathrm{C}), 606(\mathrm{C}-\mathrm{S})$ $\mathrm{cm}^{-1} .{ }^{1} \mathrm{H} \mathrm{NMR}\left(300 \mathrm{MHz} ; \mathrm{CDCl}_{3} ; \mathrm{Me}_{4} \mathrm{Si}\right) ; \delta 6.72-7.40$ $(\mathrm{m}, 7 \mathrm{H}, \mathrm{Ar}-\mathrm{H}), 5.33(\mathrm{~d}, 1 \mathrm{H}, \mathrm{CH}), 5.02(\mathrm{~s}, 1 \mathrm{H}, \mathrm{CH})$, 4.20 (d, 1H, CH), 2.29 (s, 3H, CH $\left.\mathrm{CH}_{3}\right), 2.12\left(\mathrm{~s}, 3 \mathrm{H}, \mathrm{CH}_{3}\right)$; ${ }^{13} \mathrm{C}$ NMR $\left(75 \mathrm{MHz}, \mathrm{CDCl}_{3}, \mathrm{Me}_{4} \mathrm{Si}\right): \delta 20.90\left(\mathrm{CH}_{3}\right)$, $22.52\left(\mathrm{CH}_{3}\right), 57.52(\mathrm{CH}), 71.60(\mathrm{CH}), 83.00$ (spiro C), 125.90-148.20 (Ar-C), $191.20(\mathrm{C}=\mathrm{O})$. Found: $\mathrm{C}$, 81.15; H, 5.56; S, 6.78\%. Calcd for $\mathrm{C}_{32} \mathrm{H}_{26} \mathrm{O}_{2} \mathrm{~S}: \mathrm{C}$, $80.98 ; \mathrm{H}, 5.52 ; \mathrm{S}, 6.76 \%$.

$2.5 \mathrm{c}$ Spiro $\left\{2^{\prime}\right.$ (o-pyridine)-5', $6^{\prime}$ (diphenyl)-2H-pyran4',3\}-5-methylbenzo[b] thiophene-2-one (5c): Grey solid. Yield: $50 \%$. m.p: $172-174^{\circ} \mathrm{C}$. IR (KBr), 3006$3033(\mathrm{Ar}-\mathrm{H}), 1725(\mathrm{C}=\mathrm{O}), 1608(\mathrm{C}=\mathrm{C}), 1600(\mathrm{C}=\mathrm{N})$ $610(\mathrm{C}-\mathrm{S}) \mathrm{cm}^{-1} .{ }^{1} \mathrm{H}$ NMR $\left(300 \mathrm{MHz} ; \mathrm{CDCl}_{3} ; \mathrm{Me}_{4} \mathrm{Si}\right)$; $\delta$ 6.65-7.21 (m, 7H, Ar-H), $5.65(\mathrm{~d}, 1 \mathrm{H}, \mathrm{CH}), 5.10$ $(\mathrm{s}, 1 \mathrm{H}, \mathrm{CH}), 4.26(\mathrm{~d}, 1 \mathrm{H}, \mathrm{CH}), 2.30\left(\mathrm{~s}, 3 \mathrm{H}, \mathrm{CH}_{3}\right) ;{ }^{13} \mathrm{C}$ NMR (75 MHz; $\left.\mathrm{CDCl}_{3} ; \mathrm{Me}_{4} \mathrm{Si}\right): \delta 20.13\left(\mathrm{CH}_{3}\right), 55.50$ $(\mathrm{CH}), 70.23(\mathrm{CH}), 83.44$ (spiroC), 124.60-154.70 (ArC), $190.90(\mathrm{C}=\mathrm{O})$. Found: C, 78.33; H, 5.21; N, 3.20; $\mathrm{S}, 6.81 \%$. Calcd for $\mathrm{C}_{30} \mathrm{H}_{23} \mathrm{NO}_{2} \mathrm{~S}: \mathrm{C}, 78.06 ; \mathrm{H}, 5.02 ; \mathrm{N}$, $3.03 ; \mathrm{S}, 6.95 \%$. 


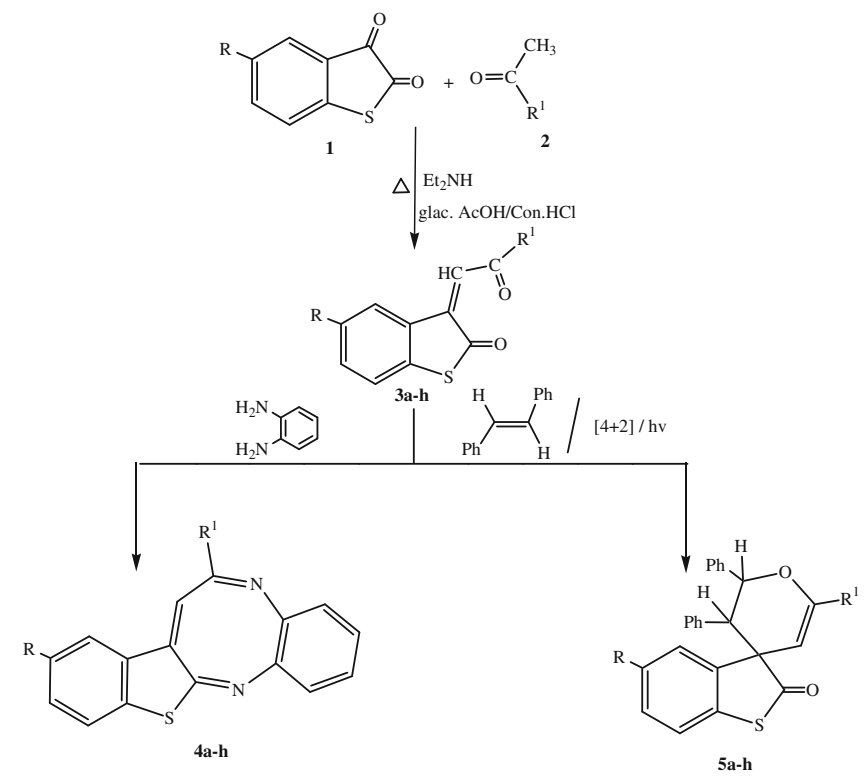

Scheme 1. Synthetic route for benzodiazocines and spiropyrans from thioisatins.

2.5d Spiro $\left\{2^{\prime}\right.$ (o-thiophene)-5', 6'-diphenyl-2H-pyran4',3\}-5-methylbenzo[b] thiophene-2-one (5d): White powder. Yield: $55 \%$. m.p: $201-204^{\circ} \mathrm{C}$. IR (KBr), 3004$3033(\mathrm{Ar}-\mathrm{H}), 1725(\mathrm{C}=\mathrm{O}), 1605(\mathrm{C}=\mathrm{C}), 612,620$ (C-S) $\mathrm{cm}^{-1} \cdot{ }^{1} \mathrm{H}$ NMR $\left(300 \mathrm{MHz} ; \mathrm{CDCl}_{3} ; \mathrm{Me}_{4} \mathrm{Si}\right) ; \delta$ 6.72-7.35 (m, 6H, Ar-H), $5.32(\mathrm{~d}, 1 \mathrm{H}, \mathrm{CH}), 4.95$ (s, $1 \mathrm{H}, \mathrm{CH}), 4.06(\mathrm{~d}, 1 \mathrm{H}, \mathrm{CH}), 2.26\left(\mathrm{~s}, 3 \mathrm{H}, \mathrm{CH}_{3}\right) ;{ }^{13} \mathrm{CNMR}$ $\left(75 \mathrm{MHz} ; \mathrm{CDCl}_{3} ; \mathrm{Me}_{4} \mathrm{Si}\right): \delta 21.9\left(\mathrm{CH}_{3}\right), 57.60(\mathrm{CH})$, $73.33(\mathrm{CH}), 82.50$ (spiro C), 127.50-144.20 (Ar-C), $191.70(\mathrm{C}=\mathrm{O})$. Found: $\mathrm{C}, 81.15 ; \mathrm{H}, 5.56 ; \mathrm{S}, 6.78 \%$. Calcd for $\mathrm{C}_{29} \mathrm{H}_{22} \mathrm{O}_{2} \mathrm{~S}_{2}$ : C, 74.65; H, 4.75; S, $13.74 \%$.
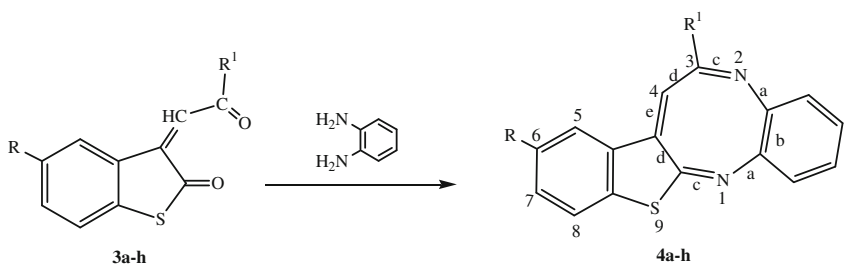

Scheme 2. Synthesis of benzodiazocines.

2.5e Spiro $\left\{2^{\prime}, 5^{\prime}, 6^{\prime}\right.$-triphenyl-2H-pyran-4',3\}-benzo[b] thiophene-2-one (5e): Light yellow solid. Yield: 56\%. m.p: $221-224^{\circ} \mathrm{C}$. IR (KBr), 3015-3044 (Ar-H), $1725(\mathrm{C}=\mathrm{O}), 1620(\mathrm{C}=\mathrm{C}), 609(\mathrm{C}-\mathrm{S}) \mathrm{cm}^{-1} \cdot{ }^{1} \mathrm{H}$ NMR (300 MHz; $\left.\mathrm{CDCl}_{3} ; \mathrm{Me}_{4} \mathrm{Si}\right) ; \delta$ 6.75-7.32 (m, 9H, Ar-H), $5.29(\mathrm{~d}, 1 \mathrm{H}, \mathrm{CH}), 4.93(\mathrm{~s}, 1 \mathrm{H}, \mathrm{CH}), 4.18(\mathrm{~d}, 1 \mathrm{H}, \mathrm{CH})$; ${ }^{13} \mathrm{C} \mathrm{NMR}\left(75 \mathrm{MHz} ; \mathrm{CDCl}_{3}, \mathrm{Me}_{4} \mathrm{Si}\right): \delta 59.55(\mathrm{CH})$, $71.33(\mathrm{CH}), 80.80$ (spiro C), 124.60-147.10 (Ar-C), $193.50(\mathrm{C}=\mathrm{O})$. Found: $\mathrm{C}, 80.81 ; \mathrm{H}, 5.56 ; \mathrm{S}, 7.10 \%$. Calcd for $\mathrm{C}_{30} \mathrm{H}_{22} \mathrm{O}_{2} \mathrm{~S}$ : C, 80.69; H, 5.25; S, 7.18\%.

$2.5 \mathrm{f}$ Spiro $\left\{2^{\prime}\right.$ (o-toluene) $5^{\prime}, 6^{\prime}$-diphenyl-2H-pyran-4',3\}benzo[b]thiophene-2-one (5f): Blackish brown solid. Yield: $58 \%$. m.p: $241^{\circ} \mathrm{C}$ (decompose). IR (KBr), 30043018 (Ar-H), $1744(\mathrm{C}=\mathrm{O}), 1611(\mathrm{C}=\mathrm{C}), 610(\mathrm{C}-\mathrm{S})$ $\mathrm{cm}^{-1} \cdot{ }^{1} \mathrm{H}$ NMR $\left(300 \mathrm{MHz} ; \mathrm{CDCl}_{3} ; \mathrm{Me}_{4} \mathrm{Si}\right) ; \delta 6.80$ $7.71(\mathrm{~m}, 8 \mathrm{H}, \mathrm{Ar}-\mathrm{H}), 5.35(\mathrm{~d}, 1 \mathrm{H}, \mathrm{CH}), 5.09(\mathrm{~s}, 1 \mathrm{H}$, $\mathrm{CH}), 4.15(\mathrm{~d}, 1 \mathrm{H}, \mathrm{CH}), 2.29\left(\mathrm{~s}, 3 \mathrm{H}, \mathrm{CH}_{3}\right) ;{ }^{13} \mathrm{C} \mathrm{NMR}$ $\left(75 \mathrm{MHz} ; \mathrm{CDCl}_{3} ; \mathrm{Me}_{4} \mathrm{Si}\right): \delta 22.60\left(\mathrm{CH}_{3}\right), 57.52(\mathrm{CH})$, $72.00(\mathrm{CH}), 83.50$ (spiro C), 124.45-145.25 (Ar-C), $190.10(\mathrm{C}=\mathrm{O})$. Found: C, 81.11; H, 5.33; S, 6.79\%. Calcd for $\mathrm{C}_{31} \mathrm{H}_{24} \mathrm{O}_{2} \mathrm{~S}$ : C, 80.84; H, 5.25; $\mathrm{S}, 6.96 \%$.

Table 1. Physical data of chalcone derivatives (3a-h).

\begin{tabular}{|c|c|c|c|c|c|c|}
\hline Entry & Product & $\mathrm{R}$ & $\mathrm{R}^{1}$ & Time (h) & M.p. $\left({ }^{\circ} \mathrm{C}\right)$ & Yield (\%) \\
\hline 1 & $3 \mathbf{a}$ & $\mathrm{CH}_{3}$ & $\mathrm{C}_{6} \mathrm{H}_{5}$ & 13 & $173-175$ & 65 \\
\hline 2 & $3 \mathbf{b}$ & $\mathrm{CH}_{3}$ & & 15 & 195-196 & 68 \\
\hline 3 & $3 c$ & $\mathrm{CH}_{3}$ & & 17 & 188 & 59 \\
\hline 4 & $3 d$ & $\mathrm{CH}_{3}$ & & 10 & $201-205$ & 70 \\
\hline 5 & $3 e$ & $\mathrm{H}$ & $\mathrm{C}_{6} \mathrm{H}_{5}$ & 14 & $210-212$ & 58 \\
\hline 6 & $3 f$ & $\mathrm{H}$ & & 10 & $156-157$ & 65 \\
\hline 7 & $3 g$ & $\mathrm{H}$ & & 13 & 180-182 & 63 \\
\hline 8 & $3 h$ & $\mathrm{H}$ & & 12 & 232 & 71 \\
\hline
\end{tabular}


Table 2. Physical data of benzodiazocine derivatives (4a-h).

\begin{tabular}{|c|c|c|c|c|c|c|}
\hline Entry & Product & $\mathrm{R}$ & $\mathrm{R}^{1}$ & Time (h) & M.p. $\left({ }^{\circ} \mathrm{C}\right)$ & Yield (\%) \\
\hline 1 & $4 \mathbf{a}$ & $\mathrm{CH}_{3}$ & $\mathrm{C}_{6} \mathrm{H}_{5}$ & 16 & 227 & 56 \\
\hline 2 & $4 b$ & $\mathrm{CH}_{3}$ & & 20 & 198-200 & 62 \\
\hline 3 & $4 c$ & $\mathrm{CH}_{3}$ & & 18 & 204-207 & 63 \\
\hline 4 & $4 d$ & $\mathrm{CH}_{3}$ & & 28 & $196(d)$ & 59 \\
\hline 5 & $4 e$ & $\mathrm{H}$ & $\mathrm{C}_{6} \mathrm{H}_{5}$ & 25 & $241(d)$ & 57 \\
\hline 6 & $4 f$ & $\mathrm{H}$ & & 21 & 203-205 & 52 \\
\hline 7 & $4 g$ & $\mathrm{H}$ & & 20 & $175-178$ & 65 \\
\hline 8 & $4 h$ & $\mathrm{H}$ & & 23 & $171-174$ & 63 \\
\hline
\end{tabular}

$2.5 \mathrm{~g}$ Spiro $\left\{2^{\prime}\right.$ (o-pyridine)-5', $6^{\prime}$ (diphenyl)-2H-pyran4',3\}-benzo[b]thiophene-2-one (5g): Brown solid. Yield: 54\%. m.p: $192^{\circ} \mathrm{C}$. IR (KBr), 3010-3040 (Ar-H), $1722(\mathrm{C}=\mathrm{O}), 1605(\mathrm{C}=\mathrm{C}), 1590(\mathrm{C}=\mathrm{N}) 610(\mathrm{C}-\mathrm{S})$ $\mathrm{cm}^{-1} .{ }^{1} \mathrm{H}$ NMR $\left(300 \mathrm{MHz} ; \mathrm{CDCl}_{3} ; \mathrm{Me}_{4} \mathrm{Si}\right) ; \delta 6.60$ $7.29(\mathrm{~m}, 8 \mathrm{H}, \mathrm{Ar}-\mathrm{H}), 5.60(\mathrm{~d}, 1 \mathrm{H}, \mathrm{CH}), 5.14(\mathrm{~s}, 1 \mathrm{H}$, $\mathrm{CH}), 4.22(\mathrm{~d}, 1 \mathrm{H}, \mathrm{CH}), 2.36\left(\mathrm{~s}, 3 \mathrm{H}, \mathrm{CH}_{3}\right) ;{ }^{13} \mathrm{C} \mathrm{NMR}$ $\left(75 \mathrm{MHz} ; \mathrm{CDCl}_{3} ; \mathrm{Me}_{4} \mathrm{Si}\right): \delta 55.65(\mathrm{CH}), 70.95(\mathrm{CH})$, 85.20 (spiro C), 126.00-153.10 ( $\mathrm{Ar}-\mathrm{C}), 192.10(\mathrm{C}=\mathrm{O})$. Found: C, 77.64; H, 4.91; N, 3.08; S, 7.20\%. Calcd for $\mathrm{C}_{29} \mathrm{H}_{21} \mathrm{NO}_{2} \mathrm{~S}$ : C, 77.83; H, 4.73; N, 3.13; S, 7.16\%.

2.5h Spiro $\left\{2^{\prime}\right.$ (o-thiophene)-5',6'-diphenyl-2H-pyran4',3\}-benzo[b]thiophene-2-one $(\mathbf{5 h})$ : Yellowish white powder. Yield: $57 \%$. m.p: $212-214^{\circ} \mathrm{C}$. IR (KBr), 3010 $3025(\mathrm{Ar}-\mathrm{H}), 1730(\mathrm{C}=\mathrm{O}), 1608(\mathrm{C}=\mathrm{C}), 612,620$ (C-S) $\mathrm{cm}^{-1} \cdot{ }^{1} \mathrm{H}$ NMR $\left(300 \mathrm{MHz} ; \mathrm{CDCl}_{3} ; \mathrm{Me}_{4} \mathrm{Si}\right) ; \delta$ 6.75-7.45 (m, 7H, Ar-H), $5.35(\mathrm{~d}, 1 \mathrm{H}, \mathrm{CH}), 4.95$ (s, $1 \mathrm{H}, \mathrm{CH}), 4.10(\mathrm{~d}, 1 \mathrm{H}, \mathrm{CH}), 2.33\left(\mathrm{~s}, 3 \mathrm{H}, \mathrm{CH}_{3}\right) ;{ }^{13} \mathrm{CNMR}$ $\left(75 \mathrm{MHz} ; \mathrm{CDCl}_{3} ; \mathrm{Me}_{4} \mathrm{Si}\right): \delta 57.60(\mathrm{CH}), 73.33(\mathrm{CH})$, 82.00 (spiro C), 130.50-142.00 (Ar-C), 192.15 $(\mathrm{C}=\mathrm{O})$. Found: $\mathrm{C}, 81.15 ; \mathrm{H}, 5.56 ; \mathrm{S}, 6.78 \%$. Calcd for $\mathrm{C}_{28} \mathrm{H}_{20} \mathrm{O}_{2} \mathrm{~S}_{2}$ : C, 74.31; H, 4.45; S, $14.17 \%$.

\section{Results and discussion}

The first step in this synthesis involved Knoevanagel condensation between the thioisatin (1) and acetophenone or its derivatives (2) in the basic medium (diethylamine) to give the aldol products, which then dehydrated easily by using con.HCl-AcOH mixture to yield the $\alpha, \beta$-unsaturated carbonyl compounds (3-phenacylidine-2-benzo[b]thiophene-2-ones) ${ }^{11}$ (3ah), commonly known as chalcones, in $58-71 \%$ yield (scheme 1). The structures of these products were established by physical (table 1) and spectral data.

The cyclocondensation reaction of appropriate 3phenacylidine-2-benzo[b]thiophene-2-ones (3a-h) with $o$-phenylene diamine in ethanol at room temperature for $21 \mathrm{~h}$ resulted in the exclusive formation of the benzodiazocine products in $52-65 \%$ yield $(\mathbf{4 a}-\mathbf{h})$ (scheme 2 ). The products were unambiguously confirmed by spectral methods. Absence of $>\mathrm{C}=\mathrm{O}$ groups and appearance of $>C=N$ groups in IR in the range of 1595$1600 \mathrm{~cm}^{-1}$, appearance of olefinic proton $(\mathrm{C}=\mathrm{CH})$ in the ${ }^{1} \mathrm{H}$ NMR spectra at $\sim 5.6 \mathrm{ppm}$ and disappearance of both $>\mathrm{C}=\mathrm{O}$ groups in ${ }^{13} \mathrm{C}$ NMR were decisively indicative of products as 3-aryl-benzo[b]thiophene[2,3d][1,2] benzodiazocine $(\mathbf{4 a - h})$. The physical data are presented in table 2 .

The photocycloaddition reaction of chalcones with trans-stilbene was carried out under nitrogen atmosphere in dry benzene with constant stirring in Heber photoreactor with medium pressure mercury arc lamp at $312 \mathrm{~nm}$. The photochemical reaction was continued until no further thioisatin was consumed (scheme 3 ).

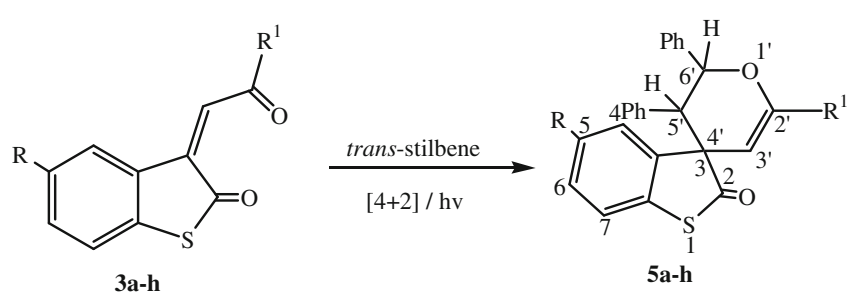

Scheme 3. Synthesis of spiropyrans. 
Table 3. Photocycloadducts (5a-h).

\begin{tabular}{|c|c|c|c|c|c|c|}
\hline Entry & Product & $\mathrm{R}$ & $\mathrm{R}^{1}$ & Time (h) & M.p. $\left({ }^{\circ} \mathrm{C}\right)$ & Yield (\%) \\
\hline 1 & $5 \mathbf{a}$ & $\mathrm{CH}_{3}$ & $\mathrm{C}_{6} \mathrm{H}_{5}$ & 24 & $221-224$ & 56 \\
\hline 2 & $5 b$ & $\mathrm{CH}_{3}$ & & 27 & 169 & 51 \\
\hline 3 & $5 c$ & $\mathrm{CH}_{3}$ & & 24 & $172-174$ & 50 \\
\hline 4 & $5 d$ & $\mathrm{CH}_{3}$ & & 21 & 201-204 & 55 \\
\hline 5 & $5 e$ & $\mathrm{H}$ & $\mathrm{C}_{6} \mathrm{H}_{5}$ & 27 & $221-224$ & 56 \\
\hline 6 & $5 f$ & $\mathrm{H}$ & & 27 & 241 & 58 \\
\hline 7 & $5 \mathrm{~g}$ & $\mathrm{H}$ & & 24 & 192 & 54 \\
\hline 8 & $5 \mathrm{~h}$ & $\mathrm{H}$ & & 21 & $212-214$ & 57 \\
\hline
\end{tabular}
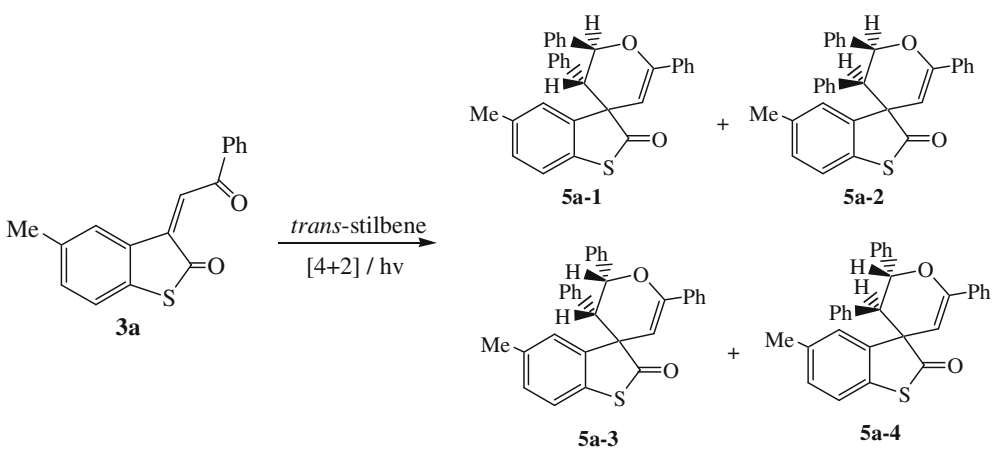

Scheme 4. Possible stereoisomers for the cycloadduct 5.

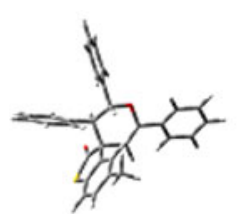

5a-1

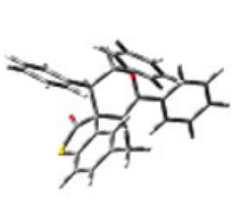

$5 a-2$

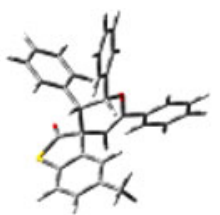

$5 a-3$

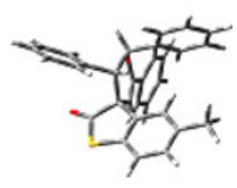

$5 a-4$

Figure 1. Optimized geometries of possible stereoisomers.

Table 4. Total energy of possible stereoisomers.

\begin{tabular}{lcccc}
\hline Stereoisomers & $\mathbf{5 a}-\mathbf{1}$ & $\mathbf{5 a - 2}$ & $\mathbf{5 a - 3}$ & $\mathbf{5 a - 4}$ \\
\hline Energy $(\mathrm{kcal} / \mathrm{mol})$ & -1094622.60 & -1094616.33 & -1094620.47 & -1094615.89
\end{tabular}




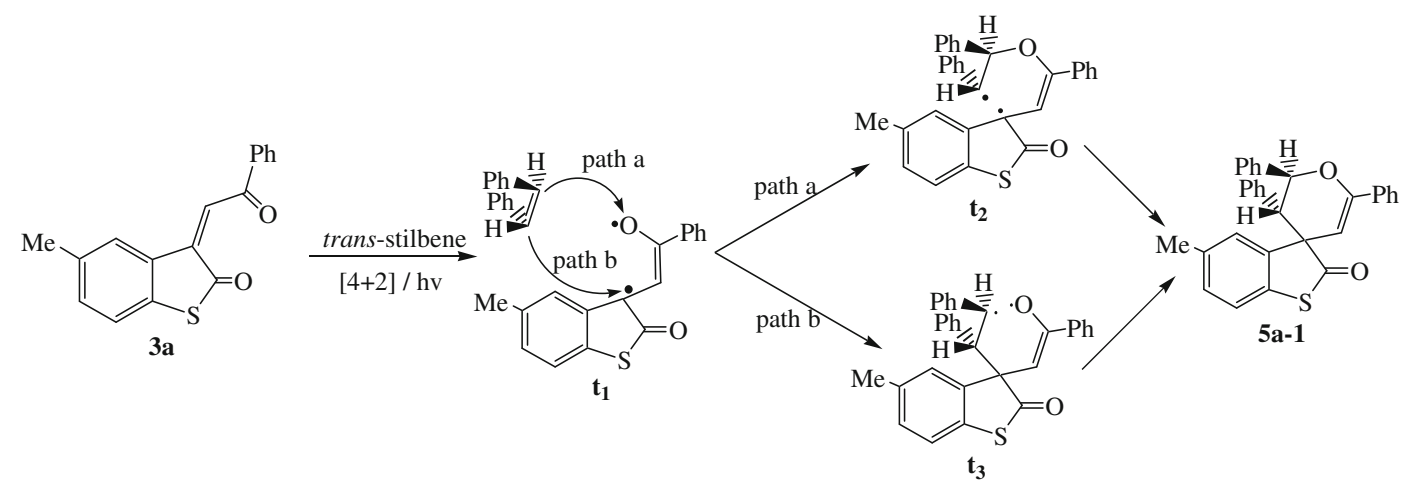

Scheme 5. Mechanism of photocycloaddition reaction.

Table 5. Energy of reactants, biradical intermediates and transition states.

\begin{tabular}{lccccc}
\hline Species & $\mathbf{3 a}$ & trans-stilbene & $\mathbf{t}_{2}$ & $\mathbf{t}_{3}$ & $\mathbf{5 a}-\mathbf{1}$ \\
\hline Energy $(\mathrm{kcal} / \mathrm{mol})$ & -755316.09 & -339300.93 & -1094575.19 & -1094568.95 & -1094622.60 \\
\hline
\end{tabular}

Subsequently, the reaction mixture was evaporated and the crude products were purified by column chromatography over silica gel to afford photo-adducts $(\mathbf{5 a}-\mathbf{h})$ in $50-58 \%$ yield (table 3 ).
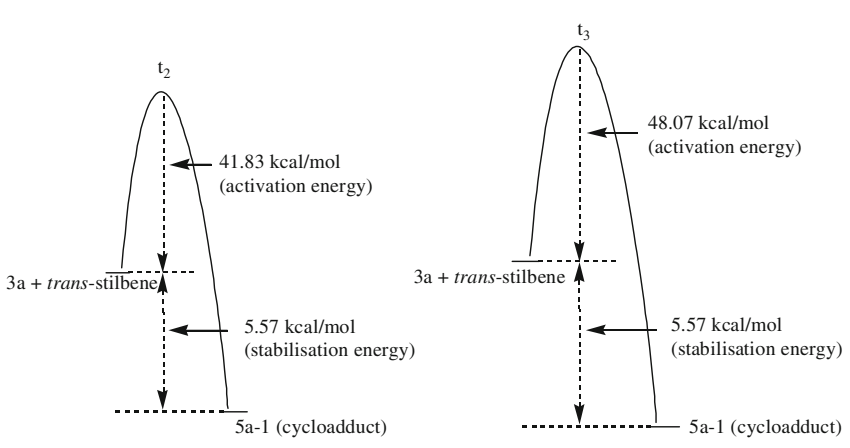

Figure 2. Energy profile diagram for the formation of cycloadduct 5a-1.
The structure of the photocycloadducts $(\mathbf{5 a}-\mathbf{h})$ has been ascertained from their spectral data. Absence of one $>\mathrm{C}=\mathrm{O}$ group in $\mathrm{IR} /{ }^{13} \mathrm{C} \mathrm{NMR}$ as well as appearance of spirocarbon at $\sim \delta 81.0 \mathrm{ppm}$ in ${ }^{13} \mathrm{C} \mathrm{NMR}$ decisively confirmed the formation of spiro $\left\{2^{\prime}, 5^{\prime}, 6^{\prime}-\right.$ triphenyl-2H-pyran-4',3\}-benzo[b] thiophene-2-one (5a-h) products.

\subsection{Regio- and stereoselectivity of photocycloaddition}

The regio- and stereoselectivity of the $[4+2]$ photocycloaddition reaction has been investigated in detail by DFT molecular orbital calculations. In this study, photocycloaddition of chalcone $\mathbf{3 a}$ with trans-stilbene is chosen as the model reaction. The attack of transstilbene on chalcone 3a may result in the formation of four isomers $\mathbf{5 a}-\mathbf{1}$ to $\mathbf{5 a}-\mathbf{4}$ (scheme 4).

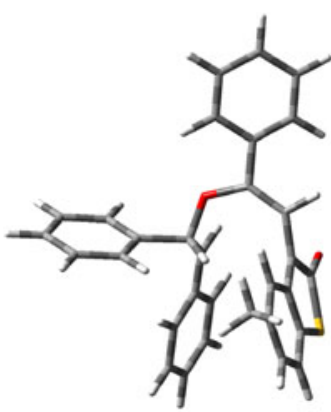

(a)

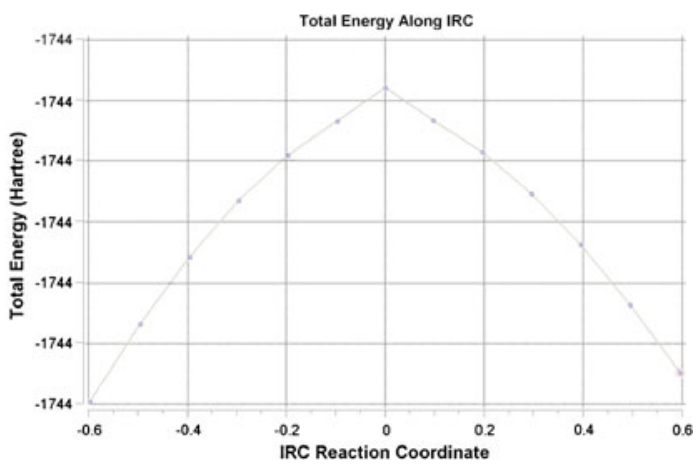

(b)

Figure 3. (a) Optimized geometry of $\mathbf{t}_{2}$, (b) IRC plot for the transition state $\mathbf{t}_{2}$. 
The geometry and energy of all four possible stereoisomers have been optimized at B3LYP/6-31G* level of theory and are shown in figure 1 . The relative energies leading to structural and stereoelectronic differences are presented in table 4 .

It is evident from table 4. that the stereoisomer (5a-1) is thermodynamically more stable than the other stereoisomers. The proximity of the bulky phenyl groups to ring system may be attributed to the relative destabilization of the remaining (5a-2 to 5a-4) stereoisomers.

\subsection{Mechanism of photocycloaddition reaction}

The next task was to illustrate a feasible mechanism for the formation of spiropyran (5a-1). A plausible mechanism is depicted in scheme 5 .

Generally, the photocycloadditions of $\alpha, \beta$ unsaturated ketones occur via triplet excited states ${ }^{11}$ and the present photocycloaddition reaction is also proposed to proceed through a triplet biradical intermediate, $\mathbf{t}_{1}$. The attack of trans-stilbene on triplet biradical $\left(\mathbf{t}_{1}\right)$ can take place via two different pathways. In path ' $\mathbf{a}$ ' the attack of trans-stilbene may take place on the oxygen radical generating intermediate triplet biradical $\mathbf{t}_{2}$ and in path ' $\mathbf{b}$ ' trans-stilbene may first attack on the carbon radical yielding another intermediate triplet biradical $\mathbf{t}_{3}$. Further, both $\mathbf{t}_{2}$ and $\mathbf{t}_{3}$ may cyclise to give the photocycloadduct 5a-1. All the ground state reactant and products and intermediate triplet biradicals as well as transition states have been optimized at B3LYP/6-31G* level to estimate their energy and to predict the feasible mechanistic pathway. The results are summarized in table 5. It is evident from these calculations that triplet radical $\mathbf{t}_{2}$ is thermodynamically more stable than $\mathbf{t}_{3}$. The extended conjugation of carbon radical with the labile $\pi$-electron pair as well as phenyl group may be attributed to its greater stability.

The potential energy barrier for reactant $\mathbf{t}_{2} \rightarrow \mathbf{5 a}-\mathbf{1}$ conversion is $41.83 \mathrm{kcal} / \mathrm{mol}$ while that of $\mathbf{t}_{3} \rightarrow \mathbf{5 a}-$ 1 conversion is $48.07 \mathrm{kcal} / \mathrm{mol}$ (figure 2). This clearly indicates that the photocycloaddition proceeds via the primary biradical $\mathbf{t}_{2}$ to yield the final cycloadduct $\mathbf{5 a - 1}$. The optimized geometry of the biradical $\mathbf{t}_{2}$ and the corresponding IRC plot for the formation of $\mathbf{5 a - 1}$ is shown in figure 3.

\section{Conclusions}

A series of novel benzodiazocine and spiropyran derivatives have been synthesized by cyclocondesation and [4 +2$]$-photocycloadditions of chalcones with $o$-phenylene diamine and trans-stilbene. The elec- tronic delocalizations, mechanism and stereochemistry of the reaction has been established theoretically by B3LYP/6-31G* method.

\section{Acknowledgements}

The financial support from the University Grants Commission (UGC) of India and for JRF fellowship from the Council of Scientific and Industrial Research (CSIR) are acknowledged.

\section{References}

1. Irie M and Uchida K 1998 Bull. Chem. Soc. Jpn. 71985

2. (a) Carrona G and Palazzo S 1968 Gazz. Chim. Ital. 98 911; (b) Tilak B D 1960 Tetrahedron. 976

3. Bremner J B and Sengpracha W 2005 Tetrahedron 61 941

4. Nowakowska Z 2007 Eur. J. Med. Chem. 42125

5. Chow Y L, Joseph T C, Quon H H and Tam J N S 1970 Can. J. Chem. 483045

6. (a) Verma S, George J, Singh S, Pardasani P and Pardasani R T 2011 Org. Med. Chem. Lett. 16 ; (b) Arora K, Singh D, Gumber A, Gupta R S, Paradasani R T and Pardasani P 2009 Heteroatom Chem. 20379

7. Abraham I, Joshi R, Pardasani P and Pardasani R T 2011 J. Braz. Chem. Soc. 22385

8. (a) Sharma G, Abraham I, Pardasani R T, Bharatam P V and Mukherjee T 2009 Bull. Chem. Soc. Jpn. 82 1477; (b) Sharma G, Abraham I, Pardasani R T, Pathak M K and Mukherjee T 2009 Res. Chem. Intermed. 35 219; (c) Pardasani R T, Pardasani P, Yadav S K and Bharatam P V 2003 J. Heterocycl. Chem. 40557

9. (a) Perrin D D, Armarego W L F and Perrin D R 1998 Purification of laboratory chemicals, 2nd ed (Oxford: Pergamon Press); (b) Vogel A I 1984 Vogel's textbook of practical organic chemistry, 4th ed (London: ELBS Longman)

10. Frisch M J, Trucks G W, Schlegel G E, Scuseria M A, Robb J R, Cheeseman J A, Montgomery Jr, Vreven T, Kudin K N, Burant J C, Millam J M, Iyengar S S, Tomasi J, Barone V, Mennucci B, Cossi M, Scalmani G, Rega N, Petersson G A, Nakatsuji H, Hada M, Ehara M, Toyota K, Fukuda R, Hasegawa J, Ishida M, Nakajima T, Honda Y, Kitao O, Nakai H, Klene M, Li X, Knox J E, Hratchian H P, Cross J B, Bakken V, Adamo C, Jaramillo J, Gomperts R, Stratmann R E, Yazyev O, Austin A J, Cammi R, Pomelli C, Ochterski J W, Ayala P Y, Morokuma K, Voth G A, Salvador P, Dannenberg J J, Zakrzewski V G, Dapprich S, Daniels A D, Strain M C, Farkas O, Malick D K, Rabuck A D, Raghavachari K, Foresman J B, Ortiz J V, Cui Q, Baboul A G, Clifford S, Cioslowski J, Stefanov B B, Liu G, Liashenko A, Piskorz P, Komaromi I, Martin R L, Fox D J, Keith T, Al-Laham M A, Peng C Y, Nanayakkara A, Challacombe M, Gill P M W, Johnson B, Chen W, Wong M W, Gonzalez C and Pople J A 2003 Gaussian 03 Revision (Pittsburgh: Gaussian, Inc.)

11. Pahup S, Anshu D and Poonam K 2010 Indian J. Chem. 49B 1135 